\title{
Crucial Components of the PEAST Algorithm in Solving Real-World Scheduling Problems
}

\author{
Nico Kyngäs, Kimmo Nurmi, and Jari Kyngäs
}

\begin{abstract}
A large number of metaheuristics and local search methods have been developed for combinatorial and global optimization. We present our PEAST algorithm which is capable of solving very difficult real-world scheduling problems, such as workforce scheduling, sports scheduling and school timetabling. The goal of this paper is to identify the crucial components of the PEAST algorithm. We believe that recognizing the importance of these components helps other researchers strengthen their population-based and local search methods.
\end{abstract}

Index Terms-PEAST algorithm, local search, population-based methods, real-world scheduling.

\section{INTRODUCTION}

A large number of metaheuristics and local search methods have been developed for combinatorial and global optimization. These methods guide the search process towards near-optimal solutions. The idea is to efficiently explore the search space with a combination of simple local search procedures and/or complex learning processes. The methods are usually non-deterministic and they may include mechanisms to avoid getting trapped in confined areas of the search space (i.e. local minima). The most celebrated methods include ant colony optimization [1], cooperative local search [2], ejection chains [3], genetic algorithms [4], hill-climbing, hyper-heuristics [5], idwalk [6], iterated local search, memetic algorithms [7], particle swarm optimization [8], simulated annealing [9], tabu search [10] and variable neighborhood search [11].

The PEAST algorithm is a population-based local search method which is capable of

1) exploring promising areas in the search space - that is, to zoom-in to find local optimum solutions to a sufficient extent - while at the same time

2) avoiding staying stuck in these areas for too long and

3) escaping from these local optima in a systematic way.

The first version of the algorithm was used to solve school timetabling problems [12]. The later versions of the algorithm have been used to solve sports scheduling problems (see e.g. [13], [14]) and workforce scheduling problems (see e.g. [15], [16]). Section II gives an outline of the PEAST algorithm and its most important components. The acronym PEAST stems from the methods used: Population, Ejection, Annealing, Shuffling and Tabu.

Manuscript received January 9, 2013; revised March 20, 2013.

Nico Kyngäs, Kimmo Nurmi, and Jari Kyngäs are with the Satakunta University of Applied Sciences, Tiedepuisto 3, Pori, Finland (e-mail: nico.kyngas@samk.fi, cimmo.nurmi@samk.fi, jari.kyngas@samk.fi).
In Section III we present three plus one real-world scheduling problems which will be used to measure the importance of the components of the PEAST algorithm. The first problem occurs in scheduling the Finnish Major Ice Hockey League [13]. The instance is derived from the 2012-2013 season for which the PEAST algorithm generated the schedule. The second problem occurs in solving the person-based multitask shift generation problem with breaks [17]. The instance is derived from the actual problems solved for a Finnish contact center. The third problem occurs in rostering drivers for transport companies [18]. The instance is derived from the biggest local transport company in Finland which uses the PEAST algorithm to optimize their driver rosters. The last problem is a school timetabling problem for which we refer to our earlier computational findings.

Section IV reports the computational results. The results identify the crucial components of the algorithm. We believe that recognizing the importance of these components helps other researchers to strengthen their population-based and local search methods.

\section{THE PEAST ALGORITHM AND ITS COMPONENTS}

The usefulness of an algorithm depends on several criteria. The two most important ones are the quality of the generated solutions and the algorithmic power of the algorithm (i.e. its efficiency and effectiveness). Other important criteria include flexibility, extensibility and learning capabilities. We can steadily note that the PEAST algorithm realizes these criteria. It has been used to solve several real-world scheduling problems and it is in industrial use. In this section we present the components of the algorithm. In Section IV we measure the importance of these components.

The PEAST algorithm is a population-based local search method. Population-based methods use a population of solutions in each iteration. The outcome of each iteration is also a population of solutions. Population-based methods are a good way to escape from local optima. The heart of the algorithm is the local search operator called GHCM (greedy hill-climbing mutation). The GHCM operator is used to explore promising areas in the search space to find local optimum solutions. Another important feature of the algorithm is the use of shuffling operators. They assist in escaping from local optima in a systematic way. Furthermore, simulated annealing and tabu search are used to avoid staying stuck in the promising search areas too long. We next discuss these and other important characteristics in detail. The pseudo-code of the algorithm is given in Fig. 1. 


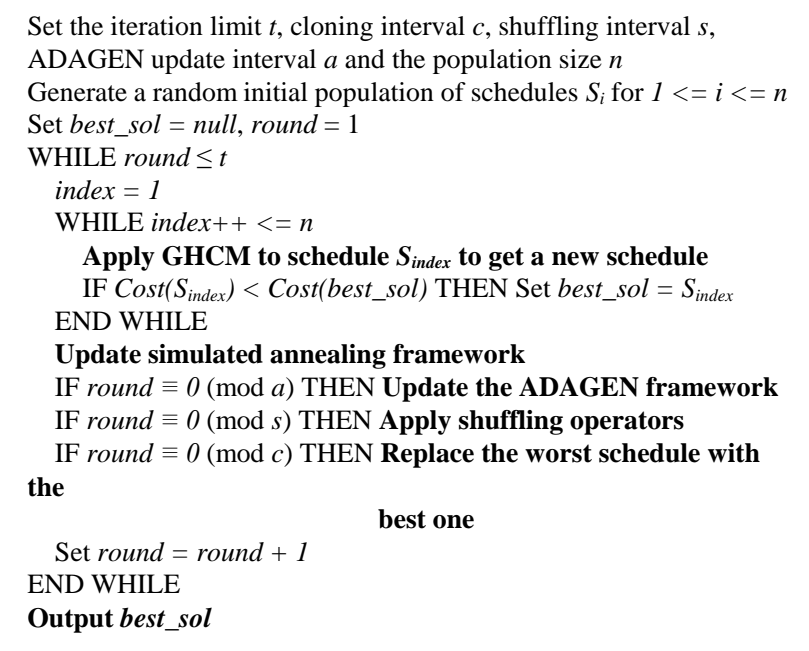

Fig. 1. The pseudo-code of the PEAST algorithm.

The GHCM operator is based on similar ideas to the Lin-Kernighan procedures [19] and ejection chains [20]. The basic hill-climbing step is extended to generate a sequence of moves in one step, leading from one solution candidate to another. The GHCM operator moves an object, $o_{1}$, from its old position, $p_{1}$, to a new position, $p_{2}$, and then moves another object, $o_{2}$, from position $p_{2}$ to a new position, $p_{3}$, and so on, ending up with a sequence of moves.

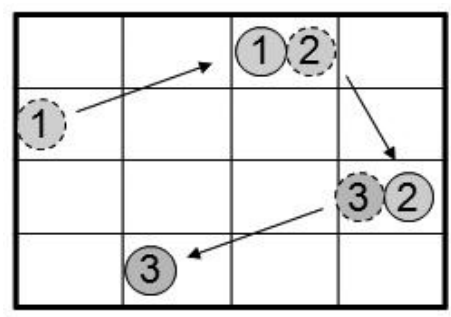

Fig. 2. A sequence of moves in the GHCM heuristic.

Picture the positions as cells as shown in Fig. 2. The initial object selection is random. The cell that receives the object is selected by considering all the possible cells and selecting the one that causes the least increase in the objective function when only considering the relocation cost. Then, another object from that cell is selected by considering all the objects in that cell and picking the one for which the removal causes the biggest decrease in the objective function when only considering the removal cost. Next, a new cell for that object is selected, and so on. The sequence of moves stops if the last move causes an increase in the objective function value and if the value is larger than that of the previous non-improving move, or if the maximum number of moves is reached. Then, a new sequence of moves is started. The maximum number of moves in the sequence is the first target we measure in Section IV.

In Section III we use the PEAST algorithm to solve real-world scheduling problems. Table I shows what cells and objects correspond to in different problems.

It may sound surprising that a good way to select the new cell for the object is to consider all possible cells and select the best one. Moreover, a good way to select a new object from that cell is again to consider all the objects in that period. Very often a not so greedy strategy ends up with better results.
Therefore, instead of selecting the first object randomly, we could use either tournament or marriage selection. In the (deterministic) tournament selection we randomly pick $k$ objects and then we choose the best one. In the marriage selection we randomly pick an object $o$, and then we try at most $k-1$ times to randomly pick a better one. We choose the first better object, or, if none is found, we choose $o$. Whether to select the first object randomly, by tournament selection or by marriage selection is the second target we measure in Section IV.

We could improve the GHCM operator by introducing a tabu list which prevents reverse order moves in the same sequence of moves. i.e. if we move an object $o$ from position $p_{1}$ to position $p_{2}$, we do not allow $o$ to be moved back to position $p_{l}$ before a new sequence of moves begins. Whether or not to use the tabu list is the third target.

TABLE I: CELLS AND OBJECTS IN THE PROBLEMS DESCRIBED IN SECTION III

\begin{tabular}{lll}
\hline \hline & CELLS & OBJECTS \\
Sports Scheduling & Game days & Games \\
Shift Generation & Shifts & Activity \\
Driver Rostering & Drivers & Shifts \\
School Timetabling & Days & Lessons \\
\hline \hline
\end{tabular}

Simulated annealing [21] is a celebrated local search heuristic which avoids staying stuck in the promising search areas too long. We could use a simulated annealing refinement to decide whether or not to commit to a sequence of moves in the GHCM operator. This refinement is different from the standard simulated annealing. It is used in a three-fold manner (see Fig. 3). Firstly, when choosing an object to be moved from a cell, a random object is chosen with probability $\exp \left(-1 / T_{k}\right)$ instead of choosing the least fit object. Secondly, when choosing the cell where to move the object, a random cell is chosen with probability $\exp \left(-1 / T_{k}\right)$ instead of choosing the fittest cell. Lastly, when the sequence of moves is cut short (i.e. a worsening move is made, and it worsens the solution more than the previous worsening move did), the whole sequence will still be committed with probability $\exp \left(-\operatorname{costDiff} / T_{k}\right)$ instead of rolling back to the best position (i.e. the position at which the objective function value is the lowest) of the sequence.

We calculate the initial temperature $T_{0}$ by

$$
T_{0}=1 / \log \left(X_{0}^{-1}\right)
$$

where $X_{0}$ is the degree to which we want to accept an increase in the cost function (we use a value of 0.75 ). The exponential cooling scheme is used to decrement the temperature:

$$
T_{k}=\alpha T_{k-1}
$$

where $\alpha$ is usually chosen between 0.8 and 0.995 . We stop the cooling at some predefined temperature. Therefore, after a certain number of iterations, $m$, we continue to accept an increase in the cost function with some constant probability, p. Using the initial temperature given above and the exponential cooling scheme, we can calculate the value 


$$
\alpha=\left(-1 /\left(T_{0} \log p\right)\right)^{1 / m}
$$

We choose $m$ equal to the maximum number of iterations and $p$ equal to 0.0015 .

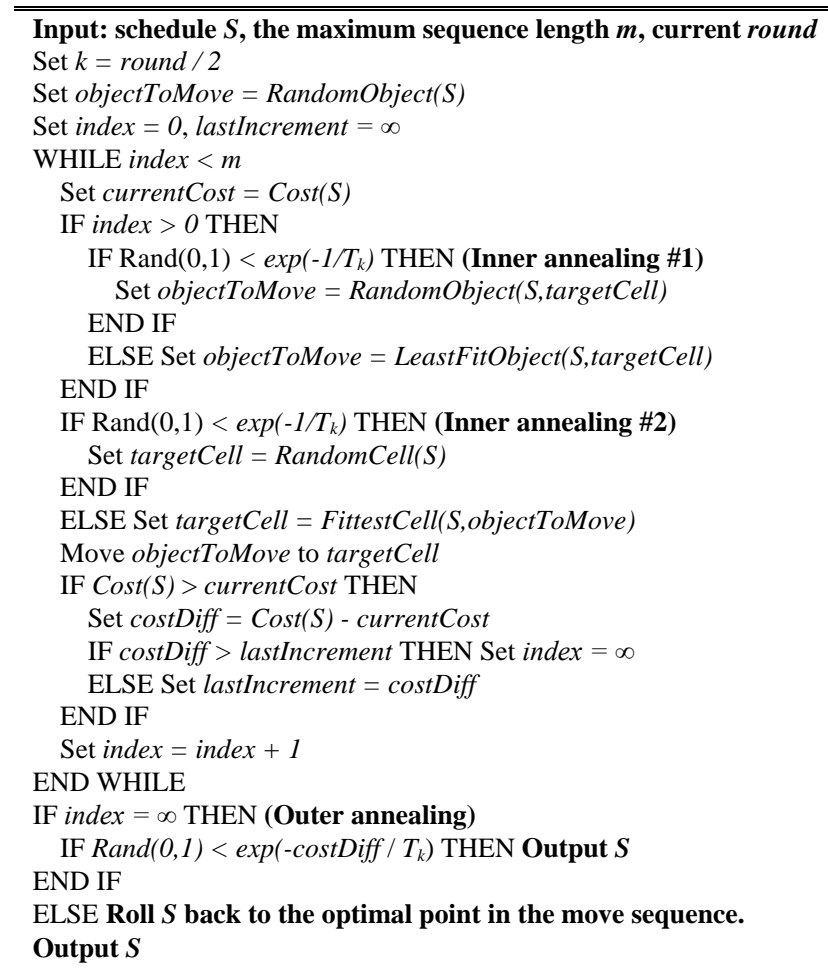

Fig. 3. The pseudo-code of a GHCM move sequence. Inner annealing allows a single move's object and destination to be non-greedy with a probability that is only dependent on the temperature of the SA scheme. Outer annealing allows a move sequence to be non-greedy with a probability that is dependent on both the current temperature and the amount by which the last move worsened the schedule.

Whether not to use the simulated annealing refinement or to use only the inner annealing or to use both inner and outer annealing is the fourth target.

For most PEAST applications we introduce a number of shuffling operators - simple heuristics used to perturb a solution into a potentially worse solution in order to escape from local optima - that are called upon according to some rule. We call the operator every $m / 20$ th iteration of the algorithm, where $m$ equals the maximum number of iterations with no improvement to the cost function. The idea of shuffling is the same as in hyperheuristics [22] but the other way around. Hyperheuristic is a mechanism that chooses a heuristic from a set of simple heuristics, applies it to the current solution to get a better solution, then chooses another heuristic and applies it, and continues this iterative cycle until the termination criterion is satisfied. We introduce a number of simple heuristics that are used to worsen the current solution instead of improving it.

Whether or not to use shuffling operators is the fifth target. Table II shows one example of shuffling operators for the real-world problems we solve in Section III.

No crossover operators are applied to the population of schedules. Every $c$ iterations the least fit individual is replaced with a clone of the fittest individual. This operation is completely irrespective of the globally fittest schedule (best_sol in Fig. 1) found. Whether to use a population of size $n$ or to run the algorithm with $n$ times the number of iterations without any population mechanism is the sixth target.

TABLE II: ONE SHUFFLING OPERATOR FOR THE PROBLEMS DESCRIBED IN SECTION III

\begin{tabular}{ll}
\hline \hline $\begin{array}{l}\text { Sports } \\
\text { Scheduling }\end{array}$ & $\begin{array}{l}\text { Select a random round and move } k_{l} \text { random games } \\
\text { from that round to random rounds }\end{array}$ \\
$\begin{array}{l}\text { Shift } \\
\text { Generation }\end{array}$ & $\begin{array}{l}\text { A random stretch of a random shift is moved to a } \\
\text { random position in another shift }\end{array}$ \\
$\begin{array}{l}\text { Driver } \\
\text { Rostering }\end{array}$ & $\begin{array}{l}\text { Select two random shifts from two random employees } \\
\text { and swap the shifts, and do this } k_{2} \text { times. }\end{array}$ \\
$\begin{array}{l}\text { School } \\
\text { Timetabling }\end{array}$ & (not in use) \\
\hline \hline
\end{tabular}

The PEAST algorithm uses ADAGEN, the adaptive genetic penalty method introduced in [12]. A traditional penalty method assigns positive weights (penalties) to the soft constraints and sums the violation scores to the hard constraint values to get a single value to be optimized. The ADAGEN method assigns dynamic weights to the hard constraints based on the constant weights assigned to the soft constraints. The soft constraints are assigned fixed weights according to their significance. This means that we are searching for a solution that minimizes the (penalty) function

$$
\sum_{i} \alpha_{i} f_{i}(x)+\sum_{i} c_{i} g_{i}(x)
$$

where

$\alpha_{i}=$ a dynamically adjusted weight for hard constraint $i$

$f_{\mathrm{i}}(x)=$ cost of violations of hard constraint $i$

$c_{\mathrm{i}}=$ a fixed weight for soft constraint $i$

$g_{\mathrm{i}}(x)=$ cost of violations of soft constraint $i$

The hard constraint weights are updated every $k$ th generation using the method given in [12]. Whether to use dynamic or fixed hard constraint weights is the seventh target.

The PEAST algorithm uses random initial solutions. Instead, we could generate the starting point in a more sophisticated way. Whether to use random or more deliberately generated initial solutions is the eighth and last target we measure in Section IV.

\section{A SET OF REAL-WORLD BENCHMARK PROBLEMS}

In this section we present three plus one real-world scheduling problems which will be used to measure the importance of the components of the PEAST algorithm presented in Section II. The first problem occurs in scheduling the Finnish Major Ice Hockey League, the second in solving the person-based multitask shift generation problem with breaks and the third in rostering drivers for transport companies. The last problem is a school timetabling problem for which we refer to our earlier computational findings.

In real-world sports scheduling, the games should be scheduled in rounds in such a way that the solution is feasible and mostly acceptable to both the league authorities and the teams. Some important requirements a sports league uses for its feasible schedule include (see [23] for detailed 
requirements):

- Every team plays exactly once in every round (if a compact schedule is required).

- A team cannot play at home on a certain day (e.g. a venue is unavailable).

- Two teams cannot play at home on the same day (e.g. they share a venue).

- A game must be pre-assigned to a certain round.

The quality of the final schedule is related to the correct optimization criteria and assignments given by the league. The league prefers to optimize many goals at the same time. Some important requests for acceptable schedules include:

- A team cannot have more than two consecutive home games.

- There must be at least $k$ rounds before two teams meet again.

- A team wishes to play most of its home games on certain weekdays.

- Two teams do not want to play at home on the same day (e.g. they are located in the same region).

Although there have been a lot of published results in the field of sports scheduling [24], practical results have been modest. Some reasons for this might be that the real-world problems are too complicated, the understanding of the optimization is not good enough from the customer's side, and that researchers tend not to be good business men or even not interested to do business. We only know of a dozen cases where the researchers have been able to close a contract with a sports league owner, see e.g. [23].

The first benchmark instance we use is derived from the 2012-2013 season of the Finnish major ice hockey league. The schedule the PEAST algorithm generated is currently in use. The Finnish major ice hockey league is the biggest sports league in Finland. It has more spectators than any other league, the players are all professionals and the media pays a lot of attention to the league.

Workforce scheduling, and all of its subcategories, have become important problems all around the world. A clear reason for this is that human resources are one of the most critical and most expensive resources for public institutions and private companies. Careful planning can lead to significant improvements both in productivity and the welfare of the staff. Besides increasing employee satisfaction, effective labor scheduling can also improve customer satisfaction.

The real-world workforce scheduling process can be classified as given in Fig. 4. Workload prediction, also referred to as demand forecasting or demand modeling, is the process of determining the staffing levels - that is, how many employees are needed for each timeslot in the planning horizon. In preference scheduling, each employee gives a list of preferences and attempts are made to fulfill them as well as possible. Shift generation is the process of determining the shift structure, along with the tasks to be carried out in particular shifts and the competences required for different shifts. Days-off scheduling deals with the assignment of rest days between working days over a given planning horizon. Resource analysis determines the compatibility of the shifts and the employees. Partitioning is a process that splits the employees and shifts into matched distinct subsets that are then rostered one at a time. It is used to handle datasets of massive scope. Staff rostering, also referred to as shift scheduling, deals with the assignment of employees to shifts. In other words, days-off scheduling deals with working days and staff rostering deals with the working times of day.

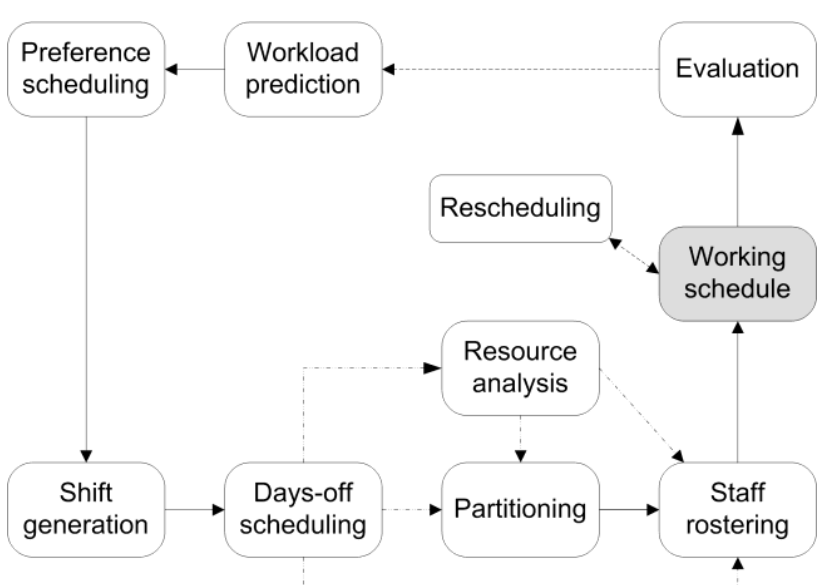

Fig. 4. The workforce scheduling process.

Rescheduling deals with ad hoc changes that are necessary due to sick leaves or other no-shows. The changes are usually carried out manually. Finally, participation in evaluation ranges from the individual employee through personnel managers to executives.

The second benchmark instance is a shift generation problem derived from the actual problems solved for a Finnish contact center [17]. The problem is to construct a set of shifts that cover the staff demand as well as possible, while satisfying a large number of constraints arising from regulatory and operational requirements and operational and employees' preferences, and ensuring that the employees can carry out the generated shifts. The most important goal is to minimize understaffing (shortage on shifts) and overstaffing (surplus on shifts). Some other important optimization goals include (see [17] for detailed criteria):

- The total workload for each task must be carried out.

- The number of shifts, i.e. the number of employees at work, must be minimized.

- The competences necessary to carry out the shifts must match the available workforce.

- Each shift must contain a given number of breaks of certain lengths, depending on the length of the shift.

- Each shift should contain at most given number of switches from one task to another.

- Each switch between tasks should occur during a break.

The third benchmark instance is derived from a problem occurring in rostering drivers for the biggest local transport company in Finland [18]. The PEAST algorithm is used to optimize their monthly driver rosters. There can be a very large number of requirements which must be met in rostering drivers. The coverage requirements ensure that there is a sufficient number of drivers on duty at all times. The regulatory requirements ensure that the driver's work contract and government regulations are respected. The operational requirements ensure that employees at work have correct competences and that they have acceptable days-off and shift structures. Some important requirements a company must consider include (see [25] for detailed requirements): 
- Employees cannot work consecutively for more than the given number of days.

- At least the given number of working days must be assigned between two separate days-off.

- An employee assigned to a late shift type must not be assigned to an early shift on the following day.

- Single days-off and single working days should be avoided.

- A balanced assignment of weekdays must be guaranteed between drivers.

- A requested day-off should be assigned.

The last benchmark problem is a school timetabling problem for which we refer to our earlier computational findings in [26]. The next section reports the computational results.

\section{Computational Results}

In this section we identify the crucial components of the PEAST algorithm using the three plus one benchmark problems described in Section III.

The tests were conducted as follows. The Standard configuration was used for getting benchmark results. Every other configuration differs from Standard in one or two parameters. In most cases only one parameter is changed, but changing certain parameters drastically affects the running time of the algorithm. In those cases the number of generations is changed in order to balance out the running time. This modification is made for configurations affecting the length of a move sequence, the usage of simulated annealing and the population size.

The first component to be tested is the maximum length of a move sequence. The tested numbers are 1 (used in configuration MS1), 5 (MS5) and 10 (Standard).

The second component to be tested is the selection method for the first object in a move sequence. The tested methods are random (Standard), tournament selection (Tour) and marriage selection (Marr). The random method chooses a completely random object among all the cells. The tournament selection method picks $S$ random objects among all the cells and chooses the one the removal of which results in the lowest cost. The marriage selection method picks a random object, and then at most $S$-1 more random objects one at a time, and chooses the first one the removal of which results in a lower cost (or the first one, in case no better moves are found). The parameter $S$ is the total number of objects divided by the total number of cells (i.e. the average number of objects per cell), and thus is dependent on the instance at hand.

The third component to be tested is the existence of a tabu list. A static length of 10 was chosen for the list. The tabu list is present in all the configurations except for NoTL.

The fourth component to be tested is simulated annealing. There are four possible configurations: both inner and outer annealing (Standard), only inner annealing (IA), only outer annealing $(O A)$ and neither inner nor outer annealing (No $S A)$. The removal of inner annealing approximately doubles the running time of the algorithm, wherefore the number of generations used in pertinent instances has been approximately halved in order to achieve equal running time.
The fifth component to be tested is shuffling operators. The shuffling operators are problem-specific, and the only trial we conduct is whether they should be used (Standard) or not (No shuffling).

The sixth component to be tested is the population size of the solution pool. This component has two possibilities: population of 10 (Standard) and population of 1 (Popl).

The seventh component to be tested is ADAGEN, the adaptive penalty system that dynamically changes the coefficients used for different constraints in the guiding function during the run of the algorithm. Three configurations were tested: normal ADAGEN (Standard), static lower bound coefficients $(L B)$ and static upper bound coefficients $(U B)$. Using static bound coefficients means that the coefficients do not change dynamically during the run. Instead, they are fixed to the values that usually define their respective lower or upper bounds in the ADAGEN system.

The eighth component to be tested is the impact of the initial solution to the end result. The standard version uses a random initial solution: each object is assigned to a random cell irrespective of the other objects. The "sophisticated" initial solution (InitSol) imposes some structure on the randomness of the initial solution; the exact method varies from problem to problem. The sports scheduling instance is initialized with Schreuder's method as described in [27]. The shift generation instance is initialized with a fast heuristic that yields a solution without hard constraint violations. The staff rostering instance is initialized without any employee having two or more shifts per day.

Table III shows the results of the sports scheduling runs. Each cell follows the format $x-y$, where $x$ is the number of hard constraint violations and $y$ is the number of soft constraint violations. The results for each configuration are first sorted by hard constraint violations and then by soft constraint violations. The quartiles are taken with respect to this ordering. The Avg column contains a combination of the average hard constraint violations and the average soft constraint violations. The table has been sorted from best to worst by each column from left to right.

TABLE III: RESULTS OF 100 RUNS OF THE SPORTS SCHEDULING INSTANCE WITH DIFFERENT PARAMETER CONFIGURATIONS

\begin{tabular}{|c|c|c|c|c|c|c|}
\hline & Avg & Min & $\mathrm{Q}_{1}$ & $\mathrm{Q}_{2}$ & $\mathrm{Q}_{3}$ & $\operatorname{Max}$ \\
\hline Marr & $1-175$ & $0-141$ & $1-160$ & $1-176$ & $2-169$ & $4-175$ \\
\hline Tour & $1-178$ & $0-147$ & $0-186$ & $1-185$ & $2-174$ & 4-159 \\
\hline No $S A$ & $3-158$ & $1-137$ & $2-163$ & $3-160$ & $4-152$ & $6-156$ \\
\hline$U B$ & $3-162$ & $1-139$ & $2-157$ & $3-154$ & 4-154 & $6-153$ \\
\hline MS5 & $3-162$ & $1-143$ & $2-164$ & $3-161$ & 4-154 & $6-159$ \\
\hline $\begin{array}{l}\text { No } \\
\text { shuffling }\end{array}$ & $3-165$ & 0-192 & $2-157$ & 3-156 & 4-159 & $8-143$ \\
\hline$L B$ & $5-153$ & $1-130$ & 4-155 & $5-156$ & $7-136$ & $9-157$ \\
\hline Standard & $6-165$ & $2-158$ & $4-181$ & 6-169 & $8-152$ & $11-170$ \\
\hline$I A$ & $6-166$ & $1-159$ & $5-149$ & $6-161$ & $7-178$ & $11-257$ \\
\hline No TL & $6-168$ & $1-174$ & $5-160$ & $7-150$ & $8-165$ & $11-187$ \\
\hline MS1 & $\begin{array}{l}13- \\
195\end{array}$ & $7-187$ & 11-194 & $13-184$ & 14-198 & $24-215$ \\
\hline Pop1 & $\begin{array}{l}15- \\
153\end{array}$ & $\begin{array}{l}10- \\
347\end{array}$ & $14-138$ & $15-142$ & $16-147$ & 18-139 \\
\hline
\end{tabular}


The results with the sophisticated initial solution were removed from the table due to the extreme drop in quality (over one hundred hard constraint violations per run). The configurations without move sequences $(M S 1)$ and a population of concurrent solutions (Popl) were also significantly worse than the other configurations, yielding no solutions with less than seven hard constraint violations. Only three configurations yielded solutions without hard constraint violations, and only one of those configurations yielded at least 25 such runs. The best gains were made by changing the way the initial object is chosen in a move sequence to a greedier version. Removing either one of the major stochastic components, i.e. simulated annealing or shuffling, also improves the results by a little margin. This indicates that removing a measure of stochasticity from the algorithm might be a good idea.

TABLE IV: RESULTS OF 100 RUNS OF THE SHIFT GENERATION INSTANCE

\begin{tabular}{|l|c|c|c|c|c|c|}
\hline & Avg & Min & $\mathrm{Q}_{1}$ & $\mathrm{Q}_{2}$ & $\mathrm{Q}_{3}$ & Max \\
\hline No TL & $0-87$ & $0-74$ & $0-81$ & $0-85$ & $0-91$ & $1-95$ \\
\hline MS5 & $0-90$ & $0-71$ & $0-83$ & $0-88$ & $0-95$ & $0-157$ \\
\hline UB & $0-90$ & $0-71$ & $0-83$ & $0-90$ & $0-96$ & $1-92$ \\
\hline InitSol & $0-90$ & $0-72$ & $0-83$ & $0-88$ & $0-96$ & $0-133$ \\
\hline LB & $0-90$ & $0-75$ & $0-83$ & $0-89$ & $0-95$ & $1-103$ \\
\hline Marr & $0-91$ & $0-69$ & $0-85$ & $0-90$ & $0-101$ & $2-93$ \\
\hline Standard & $0-91$ & $0-71$ & $0-85$ & $0-89$ & $0-97$ & $1-121$ \\
\hline IA & $0-94$ & $0-75$ & $0-87$ & $0-92$ & $0-102$ & $1-100$ \\
\hline OA & $0-99$ & $0-79$ & $0-90$ & $0-101$ & $0-128$ & $2-121$ \\
\hline Tour & $0-103$ & $0-79$ & $0-93$ & $0-102$ & $0-113$ & $1-152$ \\
\hline No SA & $2-107$ & $0-76$ & $0-101$ & $0-132$ & $1-124$ & $22-119$ \\
\hline
\end{tabular}

TABLE V: RESULTS OF 100 RUNS OF THE STAFF ROSTERING INSTANCE WITH DIFFERENT PARAMETER CONFIGURATIONS

\begin{tabular}{|l|c|c|c|c|c|c|}
\hline & Avg & Min & $\mathrm{Q}_{1}$ & $\mathrm{Q}_{2}$ & $\mathrm{Q}_{3}$ & Max \\
\hline Tour & $2-351$ & $0-3$ & $1-201$ & $2-144$ & $4-5$ & $8-38$ \\
\hline NoSA & $3-158$ & $0-5$ & $2-5$ & $3-56$ & $4-4$ & $6-3$ \\
\hline Marr & $3-170$ & $0-934$ & $2-5$ & $3-3$ & $4-3$ & $6-7$ \\
\hline No shuffling & $4-3$ & $2-2$ & $3-4$ & $4-3$ & $5-2$ & $7-3$ \\
\hline NoTabu & $4-99$ & $1-800$ & $4-4$ & $5-4$ & $6-2$ & $8-5$ \\
\hline UB & $5-96$ & $2-4$ & $4-4$ & $5-4$ & $6-4$ & $8-4$ \\
\hline MS5 & $5-114$ & $2-818$ & $4-806$ & $6-3$ & $6-615$ & $9-4$ \\
\hline Standard & $5-120$ & $1-5$ & $4-4$ & $5-3$ & $6-3$ & $8-5$ \\
\hline IA & $5-142$ & $2-5$ & $4-4$ & $5-4$ & $6-4$ & $8-5$ \\
\hline Pop1 & $8-16$ & $6-9$ & $8-6$ & $8-12$ & $9-8$ & $10-9$ \\
\hline MS1 & $18-$ & $8-$ & $16-$ & $18-$ & $19-$ & $25-$ \\
\hline & 2588 & 1987 & 2470 & 1570 & 2529 & 2997 \\
\hline
\end{tabular}

Table IV contains the results of the shift generation runs. The results are represented the exact same way as in Table III. The quartiles are taken according to this ordering. The average column contains a combination of the average hard constraint violations and the average soft constraint violations.

Certain runs are removed from the table, namely the ones with either a maximum move sequence length or population size of 1 , and those without shuffling. This is due to the lack of feasible results: not a single run with any of the aforementioned configurations ended up feasible, as opposed to all the other configurations.

Most configurations show insignificant differences. Sophisticated and random initial solutions give similar results, which is not the case in the other examined problems. However, it is obvious that both move sequence length and population size must be strictly greater than one in order to get good results. Out of the two major stochastic components of the algorithm it seems that shuffling is absolutely crucial in this particular problem. Interestingly shift generation seems to be the only problem where the removal of either has a major negative impact. We believe this is due to the fundamental structure of the problems. In the shift generation instance most hard constraint violations are caused by consecutive activities that an employee has no competence for, while the need for eight consecutive slots for any activity tends to keep the violating activities in place. This is a difficult kind of plateau that has no equivalent in the other problems.

Table V contains the results of the staff rostering runs. The results are represented as in Table III. The configuration with sophisticated initial solution was removed from the table due to an excess of constraint violations.

In this particular problem there is a lot of fluctuation in the number of soft constraint violations. This is intrinsic to the problem and the way the violations are calculated: violations in working time are often relatively large compared to all the other constraints, and they may easily dominate runs, especially ones with hard constraint violations.

It is notable that Popl and MSI are at the bottom of the table again, although only $M S 1$ is considerably worse than the other configurations. There are only three configurations that yield solutions without hard constraint violations. The best results are obtained via adding greed to the selection of the initial object in a move sequence and by reducing the stochasticity of the algorithm.

\section{CONCLUSIONS AND FUTURE WORK}

In the test problems three things led to significant drops in the quality of the results:

1) Using initial solutions that were not completely random resulted in a large number of both hard and soft constraint violations in both sports scheduling and staff rostering. In shift generation both initial solution construction methods gave almost equal results. Thus we found no evidence that a sophisticated initial solution improves results. On the contrary, random initial solutions seem to yield superior or at least as good results.

2) Making moves without compounding them into move sequences and considering these larger entities yielded significantly worse results overall than the standard algorithm.

3) Keeping track of a single solution instead of a larger population of solutions yielded results very similar to reducing move sequences to singular moves. 
We identify the associated components (random initial solution, making moves in sequences and using a population of solutions) as the crucial components of the PEAST algorithm. All the other components seem to have varying effects on the quality of the results in different problems. These results are in line with the results for school timetabling presented in [26]. However, it seems that there is room for improvement in the parameters of the algorithm. Yet we put forth that the crucial components of the PEAST algorithm are good for a broad range of real-world scheduling problems, and that they can be used to improve heuristic-based algorithms concerned with solving similar problems.

As a result of this research, we have determined that the initial object in a move sequence should be chosen more greedily using marriage selection. In the cases of sports scheduling and staff rostering we are considering reducing the stochasticity of the algorithm via the removal of the shuffling operators. However, further examination is called for.

Our next paper will compare the performance of the PEAST algorithm to that of CPLEX in real-world scheduling problems. Our preliminary findings indicate that CPLEX is better suited to solving the shift generation problem than PEAST. However, PEAST wins out in both staff rostering and sports scheduling.

\section{REFERENCES}

[1] A. Colorni, M. Dorigo, and V. Maniezzo, "Distributed Optimization by Ant Colonies," in Towards a Practice of Autonomous Systems: Proc. the First European Conference on Artificial Life, edited by F. J. Varela and P. Bourgine, Cambridge, MA: MIT Press, 1992, pp. 134-142.

[2] P. Preux and E.-G. Talbi, "Towards Hybrid Evolutionary Algorithms," International Transactions in Operational Research, vol. 6, 1999, pp. 557-570.

[3] F. Glover, "New ejection chain and alternating path methods for traveling salesman problems," in Computer Science and Operations Research: New Developments in Their Interfaces, edited by Sharda, Balci, and Zenios, Elsevier, 1992, pp. 449-509.

[4] D. E. Goldberg, Genetic Algorithms in Search, Optimization and Machine Learning, Boston, MA, USA: Kluwer Academic Publishers, 1989.

[5] E. K. Burke et al., "Hyper-heuristics: An emerging direction in modern search technology," in Handbook of Metaheuristics, edited by F. Glover and G. Kochenberger, Kluwer, 2003, pp. 457-474.

[6] B. Neveu, G. Trombettoni, and F. Glover, "Idwalk : A candidate list strategy with a simple diversification device," Lecture Notes in Computer Science, vol. 3258, 2004, pp. 423-437.

[7] P. Moscato, "On evolution, search, optimization, genetic algorithms and martial arts: Towards memetic algorithms," Technical Report 826, California Institute of Technology, Pasadena, California, 1989.

[8] R. C. Eberhart and J. Kennedy, "A New Optimizer Using Particle Swarm Theory," in Proc. the Sixth International Symposium on Micromachine and Human Science, Nagoya, Japan, 1995, pp. 39-43.

[9] S. Kirkpatrick, C. D. Gelatt Jr., and M. P. Vecchi, "Optimization by Simulated Annealing," Science, vol. 220, 1983, pp. 671-680.

[10] F. Glover, C. McMillan, and B. Novick, "Interactive Decision Software and Computer Graphics for Architectural and Space Palnning," Annals of Operations Research, vol. 5, 1985, pp. 557-573.

[11] P. Hansen and N. Mladenovic, "Variable neighbourhood search: Principles and applications," European Journal of Operations Research, vol. 130, 2001, pp. 449-467.
[12] K. Nurmi, "Genetic Algorithms for Timetabling and Traveling Salesman Problems," Ph.D. dissertation, Dept. of Applied Math., University of Turku, Finland, 1998.

[13] J. Kyngäs and K. Nurmi, "Scheduling the Finnish Major Ice Hockey League," in Proc. the IEEE Symposium on Computational Intelligence in Scheduling, Nashville, USA, 2009.

[14] J. Kyngäs and K. Nurmi, "Scheduling the Finnish 1st Division Ice Hockey League," in Proc. the 22nd Florida Artificial Intelligence Research Society Conference, Florida, USA, 2009.

[15] N. Kyngäs, D. Goossens, K. Nurmi, and J. Kyngäs, "Optimizing the Unlimited Shift Generation Problem," in Proc. the International Conference on the Applications of Evolutionary Computation, Malaga, Spain, 2012.

[16] N. Kyngäs, K. Nurmi, E. I. Ásgeirsson, and J. Kyngäs, "Using the PEAST Algorithm to Roster Nurses in an Intensive-Care Unit in a Finnish Hospital," in Proc. the 9th Conference on the Practice and Theory of Automated Timetabling (PATAT), Son, Norway, 2012.

[17] N. Kyngäs, K. Nurmi, and J. Kyngäs, "Solving the person-based multitask shift generation problem with breaks," IEEE SSCI 2013, submitted for publication.

[18] N. Kyngäs, K. Nurmi, and J. Kyngäs, "Optimizing Large-Scale Staff Rostering Instances," in Proc. the International Multiconference of Engineers and Computer Scientists, Hong Kong, 2012.

[19] S. Lin and B.W. Kernighan, "An effective heuristic for the traveling salesman problem," Operations Research, vol. 21, 1973, pp. 498-516.

[20] F. Glover, "New ejection chain and alternating path methods for traveling salesman problems," in Computer Science and Operations Research: New Developments in Their Interfaces, edited by Sharda, Balci, and Zenios, Elsevier, 1992, pp. 449-509.

[21] P. J. M. van Laarhoven and E. H. L. Aarts, Simulated annealing: Theory and applications, Kluwer Academic Publishers, 1987.

[22] P. Cowling, G. Kendall, and E. Soubeiga, "A hyperheuristic Approach to Scheduling a Sales Summit," in Proc. the 3rd International Conference on the Practice and Theory of Automated Timetabling, 2000, pp. 176-190,

[23] K. Nurmi, D. Goossens, T. Bartsch, F. Bonomo, D. Briskorn, G. Duran, J. Kyngäs, J. Marenco, C. C. Ribeiro, F. Spieksma, S. Urrutia, and R. Wolf-Yadlin, "A Framework for Scheduling Professional Sports Leagues," in IAENG Transactions on Engineering Technologies, edited by Sio-Iong Ao, USA: Springer, vol. 5, 2010, pp. 14-28.

[24] S. Knust. [2013]. Classification of Literature on Sports Scheduling. [Online]. http://www.informatik.uni-osnabrueck.de/knust/sportlit_class/

[25] E. I. Ásgeirsson, J. Kyngäs, K. Nurmi, and M. Stølevik, “A Framework for Implementation-Oriented Staff Scheduling," in Proc the 5th Multidisciplinary Int. Scheduling Conf.: Theory and Applications, Phoenix, USA, 2011

[26] K. Nurmi and J. Kyngäs, "A Framework for School Timetabling Problem," in Proc. the 3rd Multidisciplinary Int. Scheduling Conf.: Theory and Applications, Paris, France, 2007, pp. 386-393.

[27] J. Schreuder, "Constructing timetables for sport competitions," Mathematical Programming Study, vol. 13, 1980, pp. 58-67.

Nico Kyngäs was born in Raahe, Finland in 1987. He received his Ph.M degree in Mathematics from the University of Oulu in 2011. He is currently a researcher at the Satakunta University of Applied Sciences. His research interests include workforce scheduling and logistics optimization.

Kimmo Nurmi was born in Pori, Finland in 1964. He received his Ph.D degree in Applied Mathematics from the University of Turku in 1999. He is currently the research and innovation director at the Satakunta University of Applied Sciences. His research interests include workforce scheduling, sports scheduling, production line optimization and logistics optimization.

Jari Kyngäs was born in Gällstad, Sweden in 1964. He received his Ph.D degree in Computer Science from the university of Turku in 2011. He is currently a principal lecturer/researcher at the Satakunta University of Applied Sciences. His research interests include computational intelligence. 